\title{
Automatización de una planta industrial de alimentación mediante control distribuido
}

\author{
Gerardo González-Filgueira ${ }^{1}$, Francisco Javier Rodríguez Permuy ${ }^{1}$ \\ gerardo.gonzalez@udc.es, farru1980@hotmail.com \\ ${ }^{1}$ Universidade da Coruña, Escuela Politécnica Superior,15403, Ferrol, España.
}

DOI: 10.17013/risti.27.1-17

\begin{abstract}
Resumen: La logística forma parte de los procesos de automatización en la industria 4.o. De la rapidez y eficiencia con que se realicen los procesos de llenado, clasificación, empaquetado y transporte dependen la sostenibilidad industrial. La automatización de estos procesos ayuda a mejorar los ratios de eficiencia en cualquier industria. El uso de control distribuido resulta adecuado para plantas industriales del sector servicios-alimentación. como pueden ser fabricación de yogures, queso, nata, mantequilla, postres, etc. En este trabajo se presenta la automatización del proceso de fabricación de arroz con leche en una planta de productos lácteos correspondiente a una PYME. La planta objeto de automatización consta de zonas de llenado, cocción, vaciado y una zona de retorno. Para ello, se ha procedido al empleo de tres PLC's que se comunican mediante comunicación Profibus utilizando el protocolo Maestro-Esclavo. Para corroborar el correcto funcionamiento del proceso se utiliza un Sistema de Adquisición de Datos (SCADA).
\end{abstract}

Palabras-clave: Automatización, alimentación, PLC's, Profibus, SCADA.

\section{Automation of an industrial power plant through distributed control}

\begin{abstract}
Logistics is part of the automation processes in Industry 4.0. The industrial sustainability depends on the speed and efficiency with which the filling, classification, packaging and transport processes are carried out. The automation of these processes helps to improve the efficiency ratios in any industry. The use of distributed control is suitable for industrial plants in the service-food sector. such as manufacturing of yoghurts, cheese, cream, butter, desserts, etc. This paper presents the automation of the process of making rice with milk in a dairy products plant corresponding to an SME. The plant object of automation consists of areas of filling, cooking, emptying and a return zone. To do this, we have proceeded to the use of three PLC's that communicate through Profibus communication using the Master-Slave protocol. A Data Acquisition System (SCADA) is used to corroborate the correct operation of the process
\end{abstract}

Keywords: Automation, food, PLC's, Profibus, SCADA. 


\section{Introducción}

Los sistemas de automatización industrial y su funcionamiento distribuido se pueden considerar sistemas ciber-físicos (CPS: Cyber Physical Systems) (Fig.1) que forman parte de un concepto de mayor entidad, la industria 4.o (Galluscio, M. et al., 2017), encaminado a la optimización global (Prata P. et al., 2013) de las industrias (Crespo, P., , 2015) mediante la mejora de los procesos de fabricación, la reducción de costes (González M., 2015), el incremento en la calidad, la mejora de la eficiencia, la mayor flexibilidad en los procesos de producción y, en general, todos aquellos factores (Vogel-Heuser, 2018) que permitan adaptarse de manera eficiente a las necesidades del mercado al que está orientado el producto.

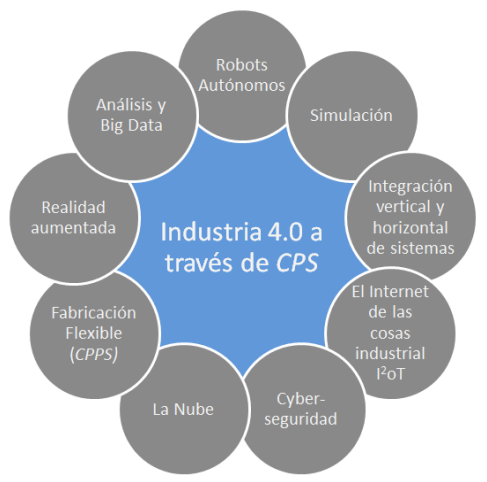

Figura 1 - Industria 4.0 a través de CPS

Los procesos de fabricación en la industria de la alimentación son susceptibles de ser automatizados para mejorar la eficiencia con el empleo de sistemas de bajo coste, bajo mantenimiento y gran durabilidad (Ali, M. S. R. and Pal, A. R., 2017). Algunas industrias de alimentación, como la fabricación de arroz con leche para postre, mantienen la manufacturación manual en la mayor parte de sus fases, pero con escasa implantación de automatización de sus procesos (Tze Ying, 2008) salvando el proceso de envasado (Fig.2).

La planta objeto de automatización, fabricación de arroz con leche para postre, consta de una zona de llenado, una zona de cocción, y una zona de vaciado y una zona de retorno. El objetivo del presente proyecto, busca una automatización integral de todo el proceso de fabricación contemplado en las zonas anteriores. Esto aporta no sólo un sentido de originalidad al proyecto, sino que implica un ahorro de costes, especialmente en momentos en los que la materia prima, en este caso el arroz, experimente un crecimiento de costes. De este modo se persigue:

- Automatizar íntegramente un proceso que tradicionalmente se estaba realizando de forma manual en alguna de sus fases, reduciendo los costes que esto conlleva.

- Reducir el tiempo de los tratamientos previos de los ingredientes, ya que éstos se realizarán de forma automática y a la vez. 
- Permitir la posibilidad de supervisión y control de todo el proceso desde un ordenador, o cualquier dispositivo táctil Tablet, Smartphone (Blech, 2015) mediante un SCADA HMI (Junior, 2003).

Para lograr los citados objetivos se parten de los siguientes requisitos:

1. El proceso de producción empleado será secuencial - por lotes, con una producción aproximada de 1000 tarrinas por lote.

2. Todo el sistema será controlado desde un panel de operario diseñado para la aplicación.

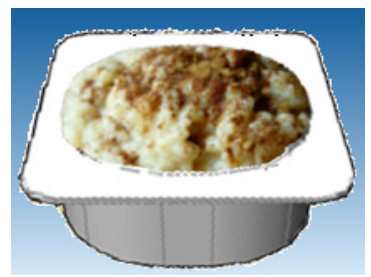

Figura 2 - Tarrina de arroz con leche

\section{Método empleado}

A la hora de implementar un sistema de control industrial se disponen de dos posibilidades: Sistema de control distribuido (DCS) y sistema de control centralizado (CCS) (Tabla 1). Esta última aproximación es empleada en el caso de sistemas poco complejos, donde un proceso puede ser gestionado directamente mediante un único elemento de control encargado de realizar todas las tareas del proceso de producción y que puede incluir un sistema de monitorización y supervisión. Conforme las necesidades de producción han requerido mayor complejidad, una tendencia ha sido la de emplear elementos de control más complejos y potentes, manteniendo en un único elemento todo el control del proceso, con la complejidad que ello supone, ya que se hace necesario hacer llegar todas las señales de sensores y cablear todos los actuadores allá donde se encuentren. Como ventajas de esta metodología se tiene que no es necesario planificar un sistema de intercomunicación entre procesos, ya que todas las señales están gestionadas por el mismo sistema. Por otro lado, para sistemas poco complejos, posee un menor coste económico. En cambio, posee numerosas desventajas, ya que si el sistema falla, toda la instalación queda paralizada, siendo necesario un sistema redundante para evitar estas situaciones. También se hace necesario el empleo de unidades de control (generalmente autómatas programables) de mayor capacidad de proceso, dada la complejidad de los problemas que debe abordar y las restricciones de tiempo límite que son habituales en los procesos industriales. Por otro lado, el cableado puede aumentar notablemente debido a las mayores distancias que pueden existir entre los sensores, actuadores y la unidad de control, aunque este problema se pueda simplificar en cierta medida debido al uso de buses de campo. 


\begin{tabular}{lll}
\hline & Control centralizado & Control distribuido \\
\hline $\begin{array}{l}\text { Aplicabilidad: procesos o } \\
\text { areas funcionales }\end{array}$ & $\begin{array}{l}\text { Sistemas poco complejos Un único } \\
\text { algorítmo: ejecución secuencial }\end{array}$ & $\begin{array}{l}\text { Sistemas complejos. Varios } \\
\text { algoritmos: ejecución en paralelo }\end{array}$ \\
\hline Gestion & elemento de control & Varios elementos de control \\
\hline Señales & $\begin{array}{l}\text { Señales gestionadas por el mismo } \\
\text { sistema }\end{array}$ & $\begin{array}{l}\text { Señales gestionadas por el } \\
\text { diferentes sistemas }\end{array}$ \\
\hline Coste económico & Pequeño & Mayor \\
\hline Seguridad en caso fallo & Se precisa Sistema redundante & Sistema redundante \\
\hline $\begin{array}{l}\text { Unidades de control } \\
\text { necesarias }\end{array}$ & $\begin{array}{l}\text { Una unidad con mayor capacidad de } \\
\text { proceso }\end{array}$ & $\begin{array}{l}\text { Varias unidades sencillas con menor } \\
\text { capacidad de proceso }\end{array}$ \\
\hline $\begin{array}{l}\text { Cableado } \\
\text { unidad de control }\end{array}$ & Menor cableado \\
\hline $\begin{array}{l}\text { Estudio de implantación } \\
\text { previo }\end{array}$ & No es necesario & Se precisa. \\
\hline Red de comunicaciones & No se precisa & Necesaria \\
\hline Error sistema & Afecta a todo el proceso & Sólo afecta a una parte del proceso \\
\hline
\end{tabular}

Tabla 1 - Sistemas industriales de control

El campo del control distribuido (García, 2004), está vinculado a procesos productivos complejos o aplicaciones industriales donde el flujo de información se puede dividir en grupos de procesos o áreas funcionales susceptibles de ser definidas por un algoritmo de control que pueda realizarse de forma autónoma (Zhu, J., Ge, Z. and Song, Z. 2017). A cada unidad se destinará un elemento de control, un autómata programable, dimensionado de acuerdo con los requerimientos del proceso considerado. En el empleo de la metodología de control distribuido es posible que cada unidad funcional consista en un proceso relativamente sencillo comparado con el proceso global, reduciendo la posibilidad de errores en la programación y permitiendo el empleo de unidades de control (autómatas programables principalmente) más sencillas y, por tanto, más económicas. Al mismo tiempo, la existencia de fallos en otras unidades de control no implica necesariamente la paralización de todos los procesos que se llevan a cabo en la planta. Como desventaja, es necesario realizar un estudio de implantación previo, ya que se deben identificar los procesos autónomos, asignar elementos a cada proceso y diseñar el modelo de intercomunicación para responder a las necesidades del proceso planteado. Debido a la interdependencia que existe entre las operaciones que tienen lugar en cada proceso, hay que tener en cuenta que es necesario interconectar los autómatas entre sí mediante entradas y salidas digitales, o a través de una red de comunicaciones para intercambio de datos y estados. Por tanto, el autómata o elemento de control evaluado debe permitir las comunicaciones. Esto permite optimizar el proceso productivo total. En esta línea se muestra el presente trabajo (Fig. 3). Para ello se han empleado tres autómatas de la serie S7 de SIEMENS utilizando el protocolo Maestro-Esclavo mediante comunicación Profibus que aporta mejores características de velocidad y tiempo de respuesta que otras topologías de red (Mahalik, 2003). 


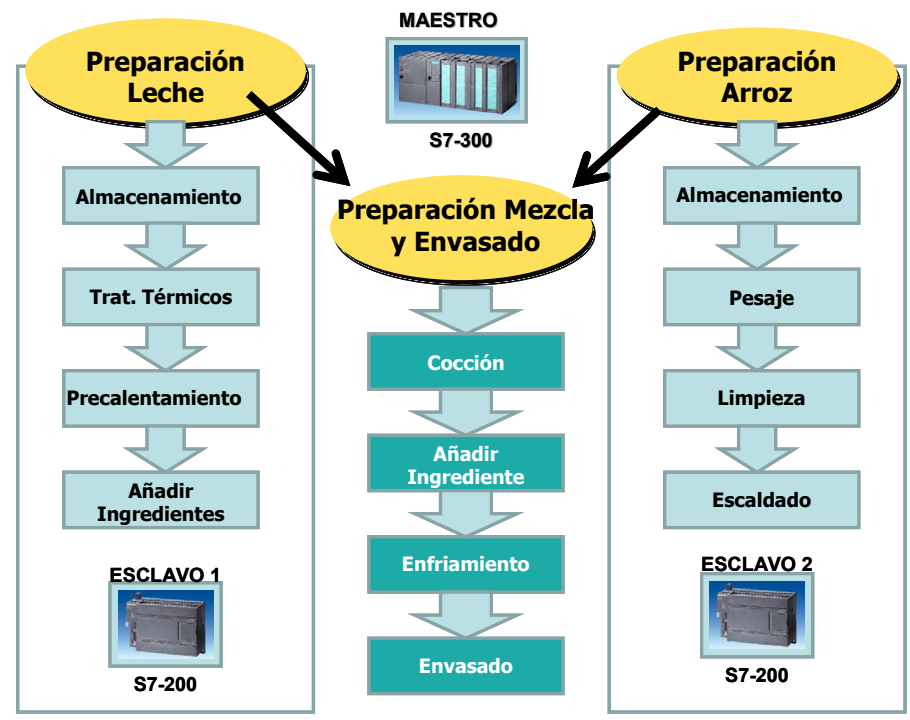

Figura 3 - Descripción General del proceso

Concretamente se emplea un S7-314-2DP como maestro y dos CPU S7-224 como unidades esclavas, junto con los módulos EM 277 PROFIBUS DP, para la comunicación Profibus de los S7-200, y los módulos de ampliación EM 223 de entradas y salidas digitales y EM 231 de entradas y salidas analógicas, que sean necesarios. El proceso de fabricación del arroz con leche [10] se ha dividido en tres grandes bloques: Bloque Arroz (controlado por una unidad S7-200), Bloque Leche (controlado por una unidad S7-200) y Bloque Mezcla y Envasado (controlado por la unidad S7-300). Dos de estos bloques, Bloque Arroz y Bloque Leche se comenzarán a ejecutar a la vez para realizar los tratamientos previos en el arroz y la leche que sean necesarios (Canales García, 2009), y a su finalización, confluyen en el tercer bloque, Bloque Mezcla y Envasado, que consistirá en el preparado de la mezcla del arroz con leche, su cocción y el posterior envasado de la misma. El proceso de producción empleado para la fabricación del arroz con leche será secuencial por lotes, con una producción aproximada de 1000 tarrinas por lote. La receta a seguir para la elaboración de este producto a nivel industrial varía de unas marcas comerciales a otras, según cada fabricante (Tabla 2). Todo el sistema podrá ser controlado desde el panel de operario diseñado para la aplicación. En cuanto al sistema SCADA de control, se ha escogido como software WinCC de SIEMENS. El SCADA se conecta a la unidad Maestra mediante conexión MPI, la cual comparte propiedades con la red Profibus y viene integrada en la CPU 314C-2DP elegida como Maestro.

Todo proceso que se pretende automatizar puede descomponerse para su análisis en dos partes: una parte operativa, que comprende las acciones que determinados elementos, como motores, cilindros neumáticos, válvulas, etc., realizan sobre el proceso; y una 


\begin{tabular}{ll}
\hline Ingrediente & Cantidad \\
\hline Arroz & 26 kilogramos \\
\hline Leche & 126 litros \\
\hline Azúcar & 25 kilogramos \\
\hline Canela en polvo & 300 gramos \\
\hline Esencia de limón & 310 gramos \\
\hline
\end{tabular}

Tabla 2 - Ingredientes para fabricación de 1 lote (1000 tarrinas)

parte de control, que programa las secuencias necesarias para la actuación de la parte operativa. De partida el sistema que se propone debe permitir:

- Reducir la presencia humana desde un único punto de control y que podrá simultanear con otros procesos.

- Sincronizar los distintos procesos que puedan existir con objeto de mejorar la eficiencia del sistema, lo que repercutirá en los costes de producción y en los niveles de calidad obtenidos en el proceso de control.

- Minimizar "errores humanos" disponiendo al operador de la información precisa y puntual acompañada de un conjunto de registros y alarmas.

La implementación del sistema de control tendrá dos aspectos fundamentales: el hardware y el software utilizado. Se busca un sistema que sea abierto, de uso general, que mediante programación pueda cumplir los requisitos necesarios para poder controlar los diferentes procesos. Por lo tanto, se garantiza el objetivo de funcionamiento de la planta.

\section{Descripción planta proceso a controlar}

El sistema de control debe gobernar todos los dispositivos que intervienen en el proceso de fabricación de arroz con leche y supervisar su correcto funcionamiento. Se ha de disponer de un programa para su funcionamiento automático y para las situaciones de emergencia, además de un sistema de control y gestión de información, apoyado en registros y alarmas que faciliten la supervisión del proceso en tiempo real y la toma de decisiones humanas si fuese necesario.

La automatización de la planta de fabricación de arroz con leche se efectuará por medio de una unidad Siemens S7-300, actuando como sistema maestro DP, y dos unidades Siemens S7-200 actuando como unidades esclavas DP. La comunicación entre las tres unidades se realizará a través de una red Profibus de periferia descentralizada (Profibus DP). Cada autómata se encargará de controlar el funcionamiento de cada uno de los tres bloques en los que se divide el proceso:

a) El Bloque Leche (Fig. 4) estará controlado por una unidad CPU-224, actuando en calidad de esclavo DP a través del módulo de ampliación EM-277, que se encargará de: 


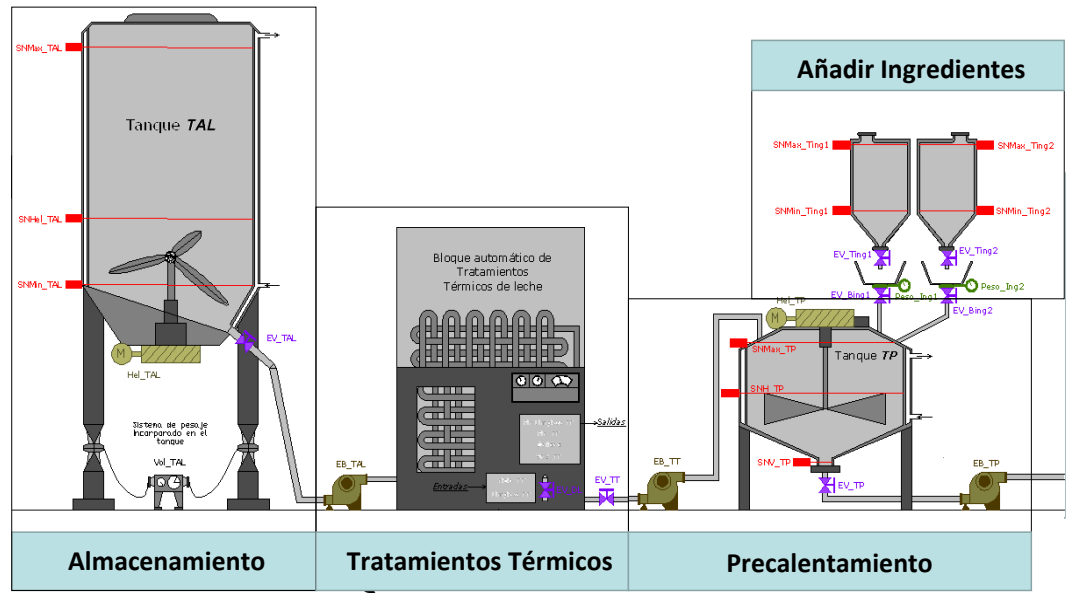

Figura 4 - Descripción del Bloque Preparación leche

- Almacenaje la leche suministrada por la central lechera.

- Tratamiento térmico de dicha leche.

- Precalentamiento.

- Adicción de ingredientes necesarios (esencia de limón y canela en polvo) antes de proceder a realizar la mezcla.

b) El Bloque Arroz (Fig. 5) estará controlado por una unidad CPU-224, actuando en calidad de esclavo DP a través del módulo de ampliación EM-277, que se ocupará de:

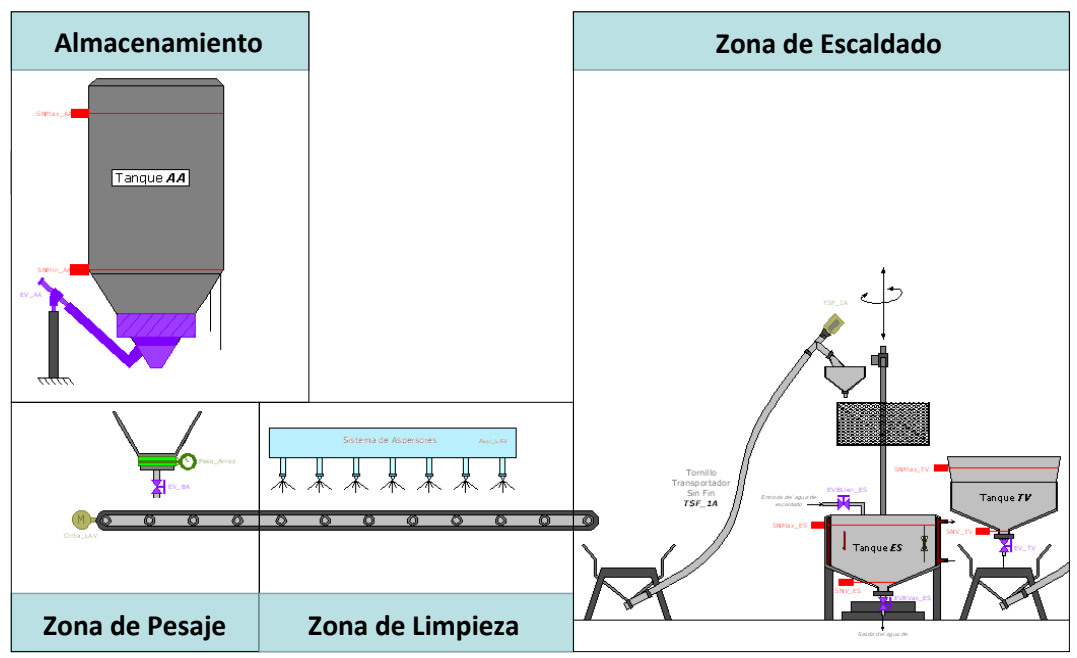

Figura 5 - Descripción del Bloque Preparación arroz 
- Almacenaje del arroz suministrado por una empresa distribuidora arrocera.

- Pesaje.

- Lavado.

- Escaldado previo a realizar la mezcla.

c) El Bloque Mezcla y Envasado (Fig.6) será el bloque principal del proceso, y estará controlado por la unidad maestra CPU 314C-2DP. Esta unidad además, será la que gobierne todo el sistema de producción, mediante su comunicación con los otros dos esclavos que se ocupará de:

- Realización de la mezcla entre el arroz escaldado y la leche precalentada.

- Realización del cocido de la mezcla.

- Adicción de los ingredientes necesarios para el producto final: azúcar y otros aromas.

- Llenado de las tarrinas.

- Termosellado.

- Etiquetado.

- Enfriamiento y almacenamiento del producto ya elaborado a espera de su distribución a los puntos de venta.

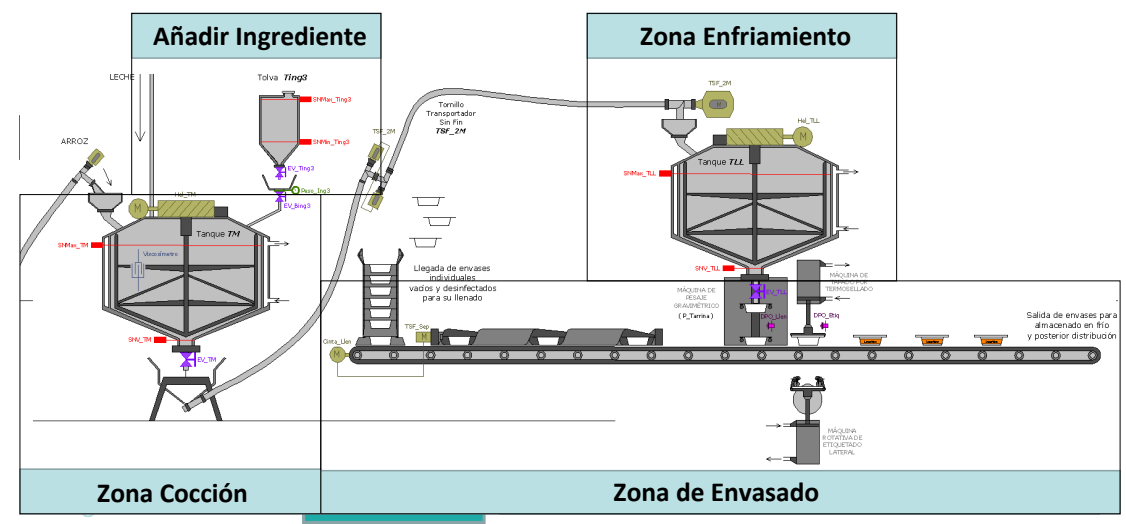

Figura 6 - Descripción del Bloque Mezcla y Envasado

\subsection{Protocolos de comunicación.}

De los bloques en los que se ha dividido el proceso, dos de ellas (preparación de la leche y preparación del arroz) transcurren de modo paralelo asíncrono y al final confluyen en una tercera (mezcla y envasado), por lo cual se concluye que se trata de un sistema distribuido (Fig. 7), adecuado para la implementar el protocolo Maestro-Esclavo.

Para la comunicación entre las unidades automáticas de control se he escogido una red PROFIBUS DP, idónea para sistemas distribuidos como es el caso de la planta a automatizar en cuestión. Debido a esto, a las dos unidades S7-200 deberán incorporarse sendos módulos de comunicación Profibus EM277 para poder conectarlas a la red 


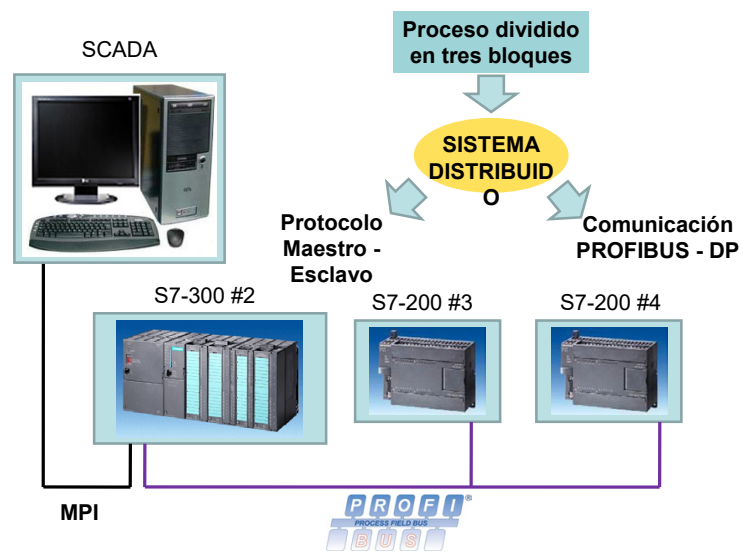

Figura 7 - Descripción Protocolos de Comunicación

gobernada por el Maestro S7-300. La red Profibus DP viene integrada en la unidad maestro S7-314-DP. En cada PLC conectado a una red Profibus, debe existir un área de memoria llamada "Imagen de proceso" o "Área de la Periferia". La imagen de Proceso para un Maestro DP está compuesta por los valores de las E/S de todos los equipos de la periferia (en el caso de que un equipo de la periferia sea un esclavo inteligente, solo se almacenarán lógicamente, las E/S que vaya a intercambiar con el maestro, no todas las

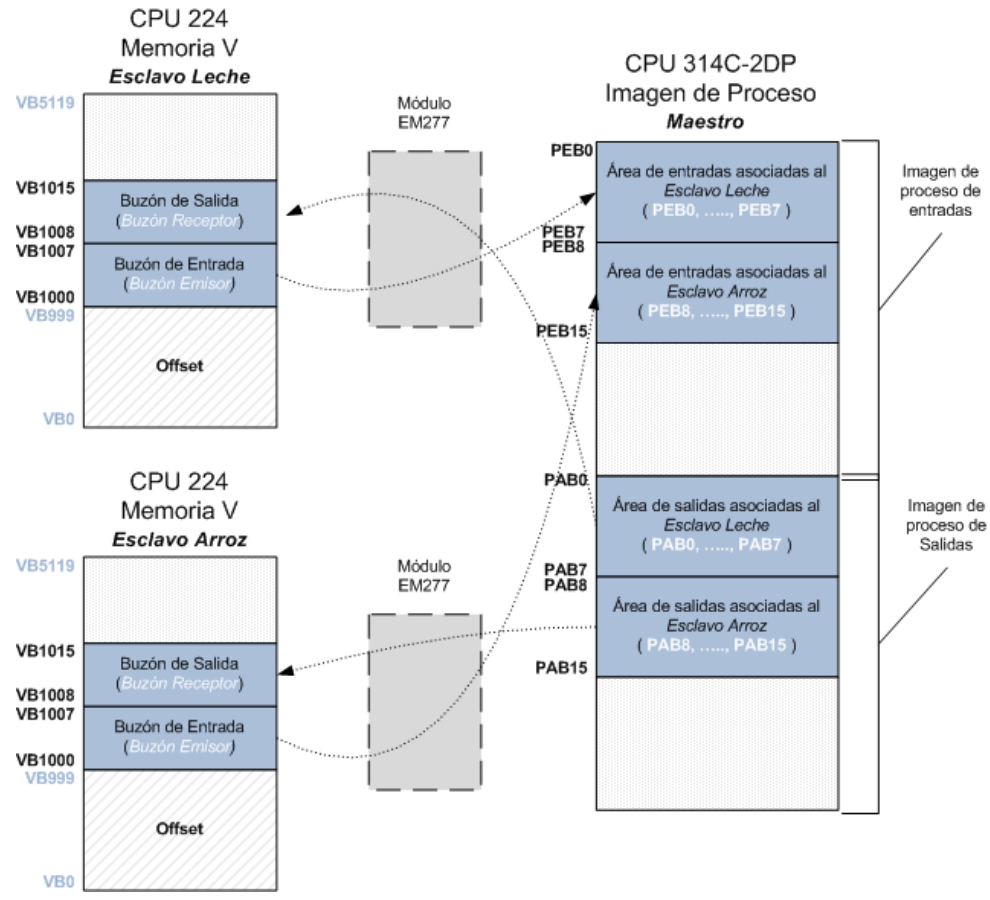

Figura 8 - Ejemplo de comunicación PROFIBUS DP 
que tenga). En cambio, para un Esclavo DP inteligente la Imagen de Proceso, constará de las entradas que vaya a enviar y de las salidas que vaya a actualizar el maestro. Como consecuencia, se sabe que en un maestro, la imagen de Proceso debería de ser lo suficientemente grande como para contener las E/S de los esclavos, y en cada esclavo, esta imagen debería ser lo suficientemente grande para contener los datos que deba recibir del maestro o enviar al maestro. En este caso, la forma de establecer la comunicación entre la CPU 314C-2DP empleada en este trabajo, y los esclavos S7-200 es muy sencilla (Fig. 8).

Se deberán definir unas áreas de memoria DP, tanto de entrada como de salida, en el momento de configurar el hardware asociado a la unidad Maestra en el Administrador SIMATIC del Software STEP 7. Se debe trabajar con dichas posiciones de memoria de la CPU 314C-2DP para comunicar las estaciones remotas. De esta forma, si se supone que la CPU 314-2DP está trabajando como Maestro y se ha configurado un Esclavo asignándole el byte de salida 4, cada vez que modifique dicho byte, se verán reflejadas dichas modificaciones en el esclavo que tiene asignada esa salida.

Lo mismo ocurre con las entradas. Si se lee una entrada que ha sido asignada a un Esclavo DP, se estará leyendo la entrada que proporciona dicho Esclavo DP. En el momento de la asignación de entradas, hay que tener claro la cantidad de datos $\left(\mathrm{n}^{\circ}\right.$ de bytes) que se quieren transmitir.

Los PLC's se interconectan mediante la red Profibus-DP, y el maestro se conecta al sistema SCADA (WinCC) mediante el cable PC Adapter (conexión MPI).

\subsection{Algorítmo del proceso}

En la automatización de una máquina es necesario prever todos los estados posibles: funcionamiento manual o semiautomático, paradas de emergencia, puesta en marcha y

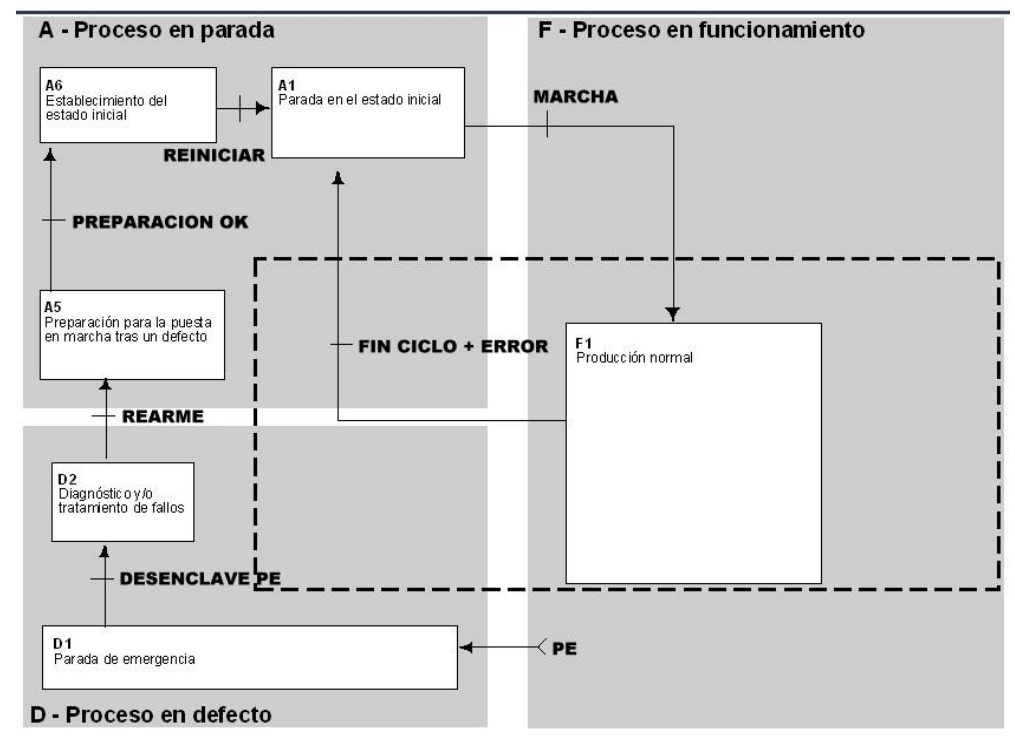

Figura 9 - Guía GEMMA del proceso 
además, el propio automatismo debe ser capaz para detectar defectos en la parte operativa y colaborar con el operario o técnico de mantenimiento para su puesta en marcha y reparación, entre otras. La guía GEMMA (Fig.9) muestra los distintos procesos que afectan a la planta.

El proceso que se describe en este trabajo es de tipo continuo. Resulta necesario disponer de un algoritmo lo más abierto posible, para poder ser implementado en diferentes PLC's, en función de las necesidades de los clientes y las capacidades del sistema. El GRAFCET de primer nivel (Fig. 10) representa el diagrama de flujo de estados del proceso. La situación de funcionamiento normal se encuentra entre los estados o y 1 del GRAFCET G1. En el momento en que se active el pulsador de parada de emergencia, sea cual sea el estado en el que se encuentre, se pasará a la etapa 2 correspondiente al estado de parada de emergencia, mediante el forzado de dicho GRAFCET. El único modo de regresar al estado de funcionamiento normal sería pasando por las etapas sucesivas de preparación tras fallo y puesta en estado inicial. Las etapas de tratamiento posterior a la emergencia, se llevarán a cabo de forma manual por parte de los operarios y personal especializado.

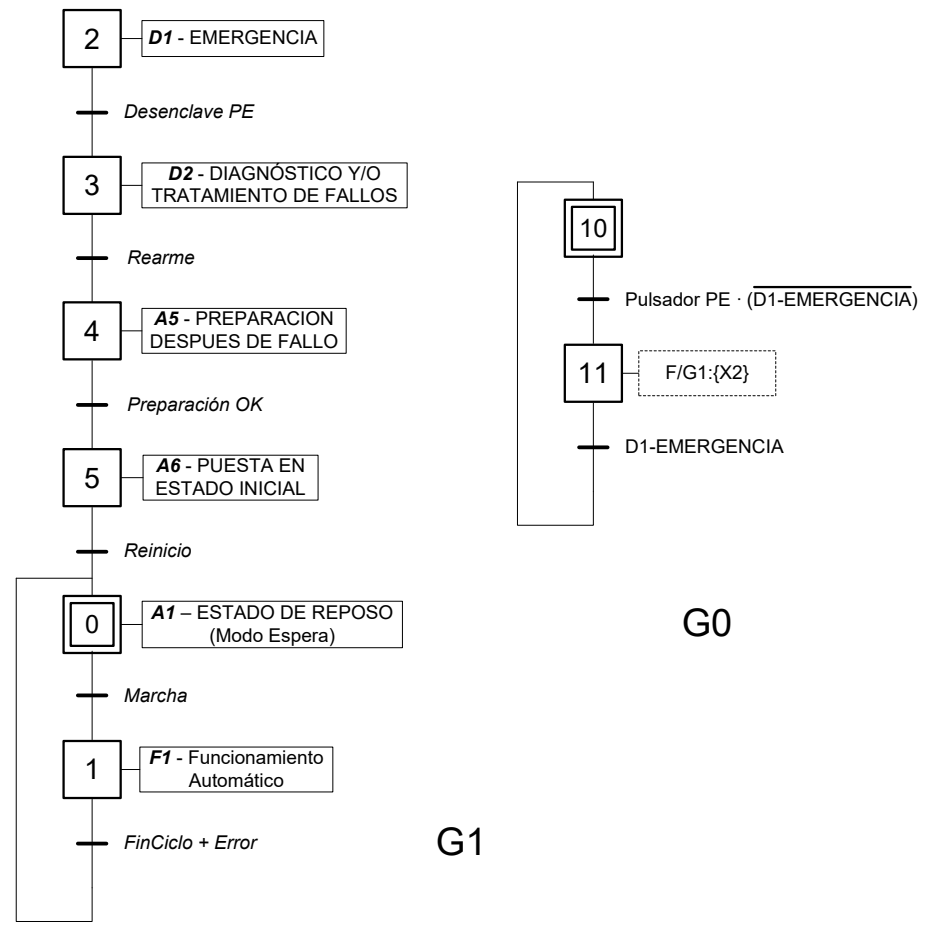

Figura 10 - GRAFCET general del proceso

En la etapa de funcionamiento automático (F1), se ha implementado el sistema de control de la planta de fabricación de arroz con leche (Fig. 11). Al igual que el GRAFCET general, esta etapa se implementará en el autómata Maestro, que es quien controla el proceso de fabricación, de modo que en determinadas situaciones se darán órdenes a los esclavos 
para que realicen las aplicaciones que tienen asignadas. Siguiendo los requerimientos del sistema indicados anteriormente, se ha implementado un programa en lenguaje de contactos que satisfaga dichas especificaciones.

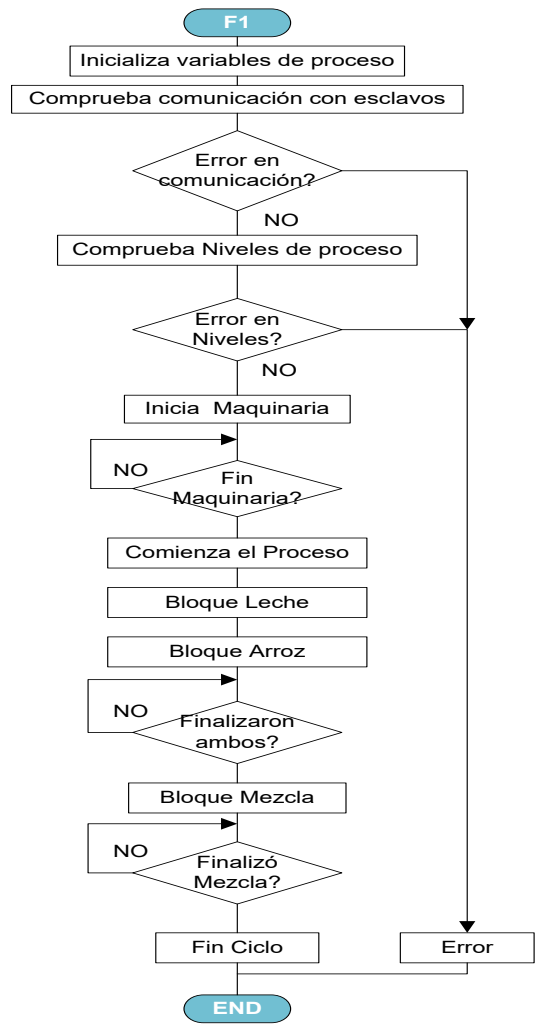

Figura 11 - Etapa F1 de funcionamiento automático

\section{Resultados}

Se precisa establecer una simulación de la planta mediante un SCADA para comprobar la correcta ejecución del sistema, y poder comparar entre la situación real y la deseada. El panel de operario es fundamental en todo proceso de automatización. En él deben disponerse los dispositivos de control, supervisión y parada de emergencia necesarios para la gestión integral del proceso. En ningún caso se considera que los sistemas SCADA sustituyen al panel de operario. Aunque haya aplicaciones gobernadas mayoritariamente por SCADA's, el panel de operario deberá existir y contener al menos el pulsador manual de parada de emergencia. En este caso se ha optado por un diseño como el mostrado en la Fig. 12.

$\mathrm{Al}$ activar el pulsador "Marcha" se inicia la producción de 1 lote (100o tarrinas de arroz con leche). El Maestro inicializa los LED's del panel encendiendo primeramente "Leche No Iniciado", “Arroz No Iniciado" y "Mezcla No Iniciado”, permaneciendo apagados el 
resto de los indicadores. Inmediatamente después se comprueba la comunicación con los esclavos y visualiza los resultados de comunicación activando los LED's que procedan en el área "COMUNICACIONES" (LED Verde= Comunicación OK, LED Rojo=Fallo en comunicación), especificando si falla la conexión con el Esclavo Leche y/o con el Esclavo Arroz. Si hay fallo de comunicación, además de encender los indicadores oportunos, se cancela la orden de producción, regresando de nuevo al estado inicial de reposo en espera de que se vuelva a activar el pulsador de MARCHA. Si no hay problema de comunicación, se pasa a comprobar niveles. El Maestro ordena a cada esclavo que compruebe el estado de los sensores de nivel de los ingredientes que están bajo el control de cada uno. Cada autómata (Maestro, Esclavo Leche y Esclavo Arroz) controla un bloque de los tres que integran el proceso de fabricación; por lo tanto controlan la maquinaria y sensores del bloque que se trate. Los esclavos tras comprobar los niveles, remiten la información al Maestro para que éste visualice los resultados en el panel de operario y se tomen las acciones oportunas en caso de error. Cada nivel a chequear dispone de dos LEDs, verde y rojo, para indicar si se alcanza o no dicho nivel respectivamente. Si no se detecta nivel mínimo en alguno de los ingredientes comprobados, se encenderá su LED rojo y el sistema cancelará la orden de producción, regresando al estado inicial en espera de que se reponga el ingrediente y se vuelva a pulsar MARCHA. Cuando todos los niveles sean correctos se pasa a inicializar la maquinaria de cada bloque y se comienza el proceso.

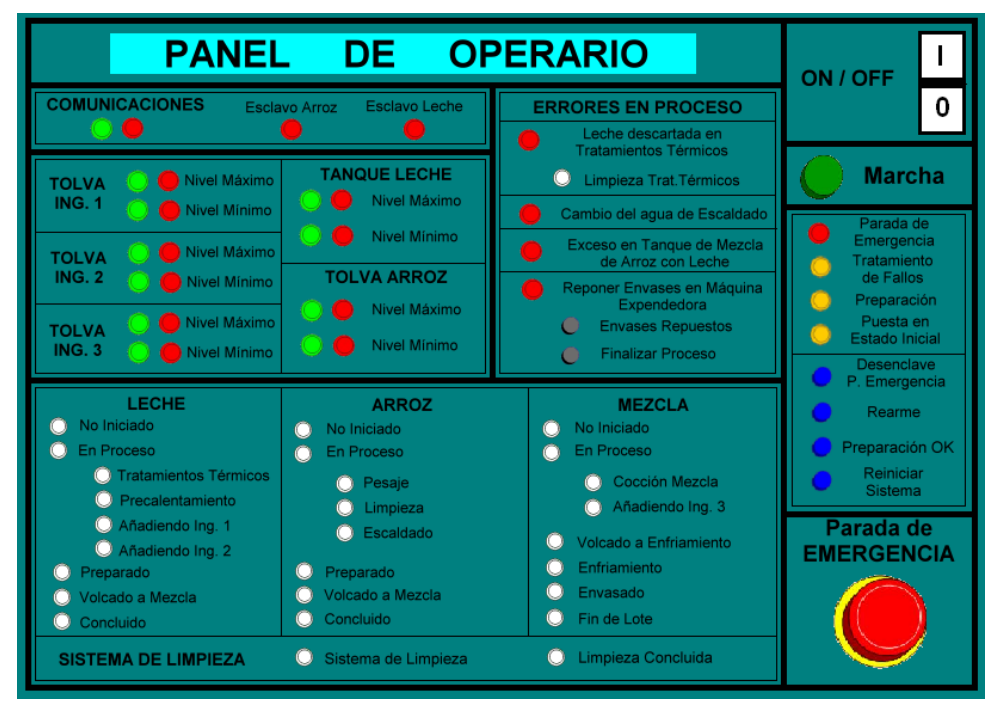

Figura 12 - Panel del operario

En primer lugar se iniciará el Bloque Leche. La leche comienza su preparación para la mezcla (Fig. 13), y el Esclavo Leche enviará constantemente información de la fase en la que se encuentra la leche, para que el Maestro actualice los indicadores del panel de operario. Cada bloque "en Proceso" consta de varias etapas. La secuencia de funcionamiento normal sería pasar a los tratamientos térmicos (y se encendería el LED “Tratamientos Térmicos" a la vez que permanece activado el LED "En Proceso") y si la 
leche no supera con éxito los tratamientos térmicos se activará el LED “Leche Descartada en Tratamientos Térmicos" indicativo de error en proceso.

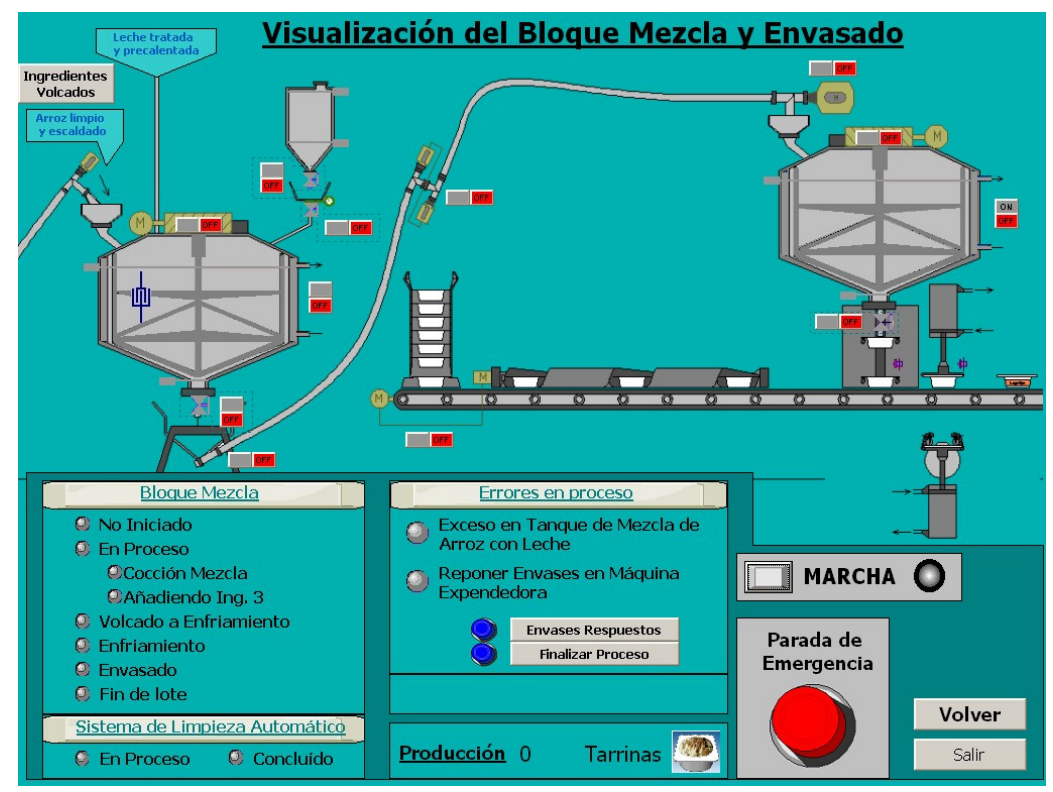

Figura 13 - Bloque de Mezcla y envasado

A continuación (Fig. 14) se pasa a la fase de Precalentamiento. Simultáneamente se da la orden al Esclavo Arroz de comenzar su aplicación, por lo que se activará el LED "Arroz En Proceso". El bloque arroz puede originar un error si el agua de escaldado del arroz no cumple con el nivel de higiene y salubridad medido por medio de su turbidez. A medida que vayan transcurriendo las fases de cada bloque, se irán actualizando los LED’s, hasta que se llegue a tener activos a la vez "Arroz Preparado" y "Leche Preparado", (al activarse los indicadores "Preparado" se desactivan los LED’s “En Proceso”), momento en el que el Maestro da la orden de trasvasar la leche y el arroz hacia el tanque de mezcla para iniciar la cocción.

Durante el tiempo que transcurra en trasvasarse la leche y volcarse el arroz al tanque de cocción, permanecerán encendidos los LED’s "Volcado a Mezcla” y cuando se finalice se activará "Concluido". En este momento, comienza el bloque Mezcla, activándose el LED “en Proceso" (y desactivándose, por tanto, el LED “No iniciado”), junto con el LED "Cocción Mezcla" para indicar que se ha iniciado la cocción conjunta del arroz con la leche. Transcurridos 20 minutos desde que se inicia la cocción, se añadirá el ingrediente 3 (azúcar o edulcorante), encendiéndose a su vez, el LED “Añadiendo Ing. 3”. En el instante en el que se alcanza la viscosidad idónea para las propiedades del producto final, se parará la cocción y se trasvasará la mezcla a la zona de enfriamiento. Durante 5 minutos, se procederá al enfriamiento del producto a una temperatura de $17^{\circ} \mathrm{C}$ en el tanque TLL (se activa el LED "Enfriamiento"). Transcurrido el tiempo de enfriado, se procede 
a llenar las tarrinas en la etapa de envasado. La etapa de envasado, incluye el proceso de llenado de envases, su termosellado y posterior etiquetado lateral. Llegado a este punto se ha finalizado la producción, y el maestro activa el sistema de limpieza automática del proceso (se activa el LED "Sistema de limpieza"). Una vez haya concluido el sistema de limpieza, el maestro actualizará el panel de operario encendiendo el LED de "Limpieza Concluida”. El sistema estará preparado para la producción del siguiente lote.

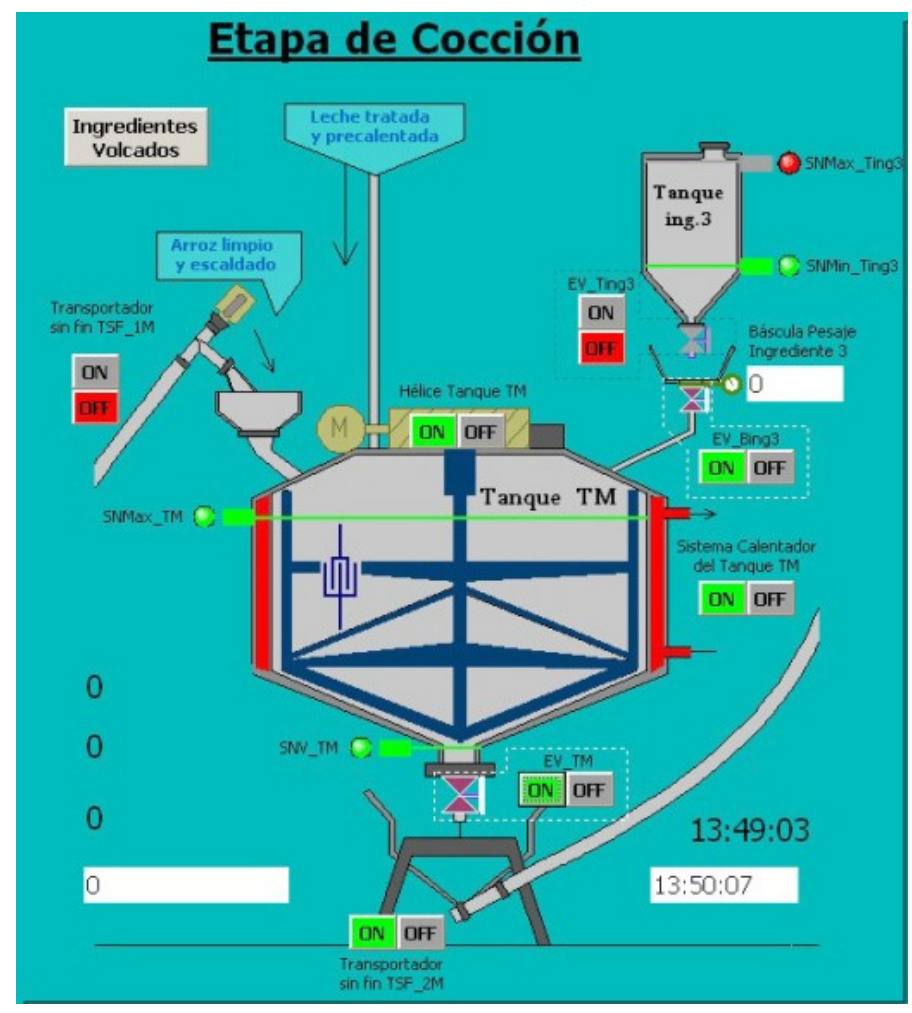

Figura 14 - Detalle etapa de cocción

\section{Conclusiones}

En este artículo se ha presentado un sistema de automatización para la automatización de fabricación de arroz con leche. En el diseño del presente sistema, se ha conseguido la creación de un sistema de control que permite gobernar todos los dispositivos que intervienen en el proceso de fabricación del arroz con leche. El sistema propuesto presenta un conjunto de ventajas como son un diseño simple, una actualización rápida de datos, fuerte rendimiento en tiempo real, bajo costo, facilidad de mantenimiento, y excelente extensibilidad. Dicho sistema constituye una herramienta fundamental para garantizar un alto porcentaje de disponibilidad y fiabilidad, además de conseguir un seguimiento en tiempo real del conjunto. Se ha implementado un sistema de supervisión y control a tiempo real que permita al operario interactuar con el proceso 
(Panel de operario). Además existe la posibilidad de implementar un control SCADA paralelo al panel de operario, sin necesidad de modificar la programación. De este modo se consigue dotar el sistema de una buena fiabilidad, adaptarse a futuras necesidades de producción del mercado. Además se ha llevado a cabo una producción secuencial por lotes, en la que se puede ajustar el número de tarrinas que contiene cada lote según las necesidades de mercado proporcionadas mediante análisis de big data, y en función del volumen de los tanques empleados. Por último, además de la parada de emergencia, imprescindible en todo proceso industrial, se ha logrado un tratamiento automático de una serie de errores, que pueden solucionarse sin la intervención el operario.

\section{Referencias}

Ali, M. S. R., \& Pal, A. R., (2017). Multi machine operation with product sorting and elimination of defective product along with packaging by their colour and dimension with speed control of motors. In Proceedings of 2017 International Conference on Electrical, Electronics, Communication, Computer, and Optimization Techniques, (pp. 1-6), Mysuru, 2017. doi: 10.1109/ICEECCOT.2017.8284575.

Blech, J. O. et al., (2015). Efficient incident handling in industrial automation through collaborative engineering. In Proceedings of 2015 IEEE 20th Conference on Emerging Technologies \& Factory Automation (ETFA), (pp. 1-8), Luxembourg. doi: 10.1109/ETFA.2015.7301533.

Canales García, E. (2009). Optimización de la formulación de arroz con leche deshidratado. Tesis Doctoral. México: Instituto Tecnológico de Durango

Crespo, P., \& Santos, V., (2015). Construção de Sistemas Integrados de Gestão para Micro e Pequenas Empresas. RISTI - Revista Ibérica de Sistemas e Tecnologias de Informação, (15), 35-49. doi: 10.17013/risti.15.35-49.

Galluscio, M. et al., (2017). A first empirical look on internet-scale exploitations of IoT devices. In Proceedings of 2017 IEEE 28th Annual International Symposium on Personal, Indoor, and Mobile Radio Communications (PIMRC), (pp. 1-7), Montreal, QC. doi: 10.1109/PIMRC.2017.8292628.

García, J., Palomo, F.R., Luque, A., Aracil, C., Quero, J.M., Carrion, D., Gamiz, F., Revilla, P., Perez-Tinao, J., Moreno, M., Robles, P., \& Franquelo, L.G., (2004). Reconfigurable distributed network control system for industrial plant automation. IEEE Transactions on Industrial Electronics, 51(6), 1168-1180. doi: 10.1109/ TIE.2004.837871.

González M., \& González, L. (2015). La co-creación como estrategia para abordar la gobernanza de TI en una organización. RISTI - Revista Ibérica de Sistemas e Tecnologias de Informação, (15), 1-16. doi: 10.17013/risti.15.1-16.

Junior, W. P., \& Pereira, C. E. (2003). A supervisory tool for real-time industrial automation systems. In Proceedings of Sixth IEEE International Symposium on Object-Oriented Real-Time Distributed Computing, (pp. 230-237), 2003. doi: 10.1109/ISORC.2003.1199258. 
Mahalik, N. P., \& Michalk, R., (2003). Retrofitting fieldbus technology in food industry. In Proceedings of 2008 World Automation Congress, (pp. 1-5), Hawaii, HI, 2008.

Prata, P., Fazendeiro, P., Augusto, C., Azevedo, S., \& Cruz-Machado, V. (2013). Ambiente Colaborativo para Avaliação de Cadeias de Abastecimento. RISTI - Revista Ibérica de Sistemas e Tecnologias de Informação, (12), 1-15. doi: 10.4304/risti.12.1-15.

Tze, Y. S., Fang, L., \& Vogel-Heuser, B., (2008). Modules, version and variability management in automation engineering of machine and plant manufacturing. In Proceedings of 2008 IEEE International Conference on Emerging Technologies and Factory Automation, (pp. 46-49), Hamburg, 2008. doi: 10.1109/ ETFA.2008.4638369.

Vogel-Heuser, B., Fischer, J., Feldmann, S., Ulewicz, S., \& Rösch, S. (2018). Modularity and architecture of PLC-based software for automated production systems: An analysis in industrial companies (journal-first abstract). In Proceedings of 2018 IEEE 25th International Conference on Software Analysis, Evolution and Reengineering (SANER), (pp. 473-473), Campobasso. doi: 10.1109/SANER.2018.8330239.

Zhu, J., Ge, Z., \& Song, Z. (2017). Distributed Parallel PCA for Modeling and Monitoring of Large-Scale Plant-Wide Processes With Big Data. IEEE Transactions on Industrial Informatics, 13(4), 1877-1885. doi: 10.1109/TII.2017.2658732. 